\title{
Solución del problema de Dirichlet mediante el método de Schwartz
}

\author{
A. Camacho Galván \\ Facultad de Ingeniería - Instituto de Geofisica, UNAM \\ Email:abel@servidor.unam.mx
}

(recibido: octubre, 1997; aceptado: diciembre, 1997)

Resumen

Se presenta un método alternativo para resolver el problema de Dirichlet para la ecuación de Laplace en dominios compuestos aplicando el método de Schwartz. Primeramente, se da a conocer un caso particular que sirve para llevar a cabo la demostración de existencia de la solución del problema propuesto; posteriormente, se expresan las condiciones suficientes para extender el criterio a otro tipo de dominios. Por último, se describe una condición geométrica, útil y de fácil comprobación, de la cual se sigue el principio de Schwartz.

\section{Abstract}

An alternative method for the resolution of the Dirichlet problem for the Laplace equation in compound domains applying the Schwartz method is shown. First, a particular case of study demoustrates the existence of the proposed problem solution; then, enough conditions for extending the criteria to another type of domains are expressed. Finally, a useful geometric condition of easy verification is described, which the Schwartz principle is followed.

Existencia de la solución

Sea un cierto dominio $G$, mismo que se descompone en tres subdominios $G_{1}, G_{2}$ y $G_{3}$, tal y como se muestra en la figura 1 .

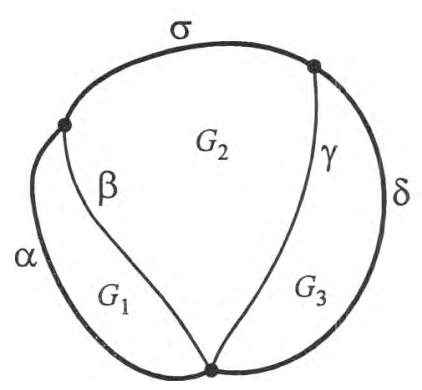

Figura 1. La partición del dominio $G$

Sea, además, $G_{1,2}$ la unión de los dominios $G_{1}, G_{2}$ y su frontera común $\beta$, es decir:

$$
G_{1,2}=G_{1} \cup G_{2} \cup \beta \text {. }
$$

Análogamente:

$$
G_{2,3}=G_{2} \cup G_{3} \cup \gamma
$$

Se observa que la frontera de la región $G_{1,2}$ está compuesta por los arcos $\alpha, \gamma$ y $\sigma$, mientras que la frontera de la región $G_{2,3}$ la constituyen los $\operatorname{arcos} \beta, \delta$ y $\sigma$.

Criterio de Schwartz 1. Se dice que el sistema de dominios $G_{1}, G_{2}$ y $G_{3}$ delimitados por los $\operatorname{arcos} \alpha, \beta, \gamma, \delta$ y $\sigma$ satisface el criterio de Schwartz, si se verifican las condiciones siguientes:

1. Cualquier función armónica en $G_{1,2}$ y continua en $\bar{G}_{1,2}$, igual a 0 sobre $\alpha \cup \sigma$ y cuyo módulo no sea mayor que 1 sobre $\gamma$, tiene un valor absoluto y no supera algún $\theta(\theta<1)$ sobre todo el arco $\beta$.

2. Cualquier función armónica en $G_{2,3}$ y continua en $\bar{G}_{2,3}$, que se anule sobre $\delta \cup \sigma$ y cuyo módulo no supere a 1 sobre $\beta$, tiene un módulo no mayor que $\theta<$ 1 sobre el arco $\gamma$.

Nota: En este criterio se supone que la constante $\theta$ puede ser escogida para el sistema dado de dominios $G_{1}, G_{2}$ y $G_{3}$, 
una vez y para siempre; en consecuencia, no depende de la función armónica considerada.

\section{Definición 1. Existencia de la solución}

1. Se dice que el dominio $G_{1,2}$ satisface la condición de existencia de solución, si para cualquier función de frontera, continua sobre $\overline{\alpha \cup \gamma \cup \sigma}$, existe una solución continua en $\overline{G_{1,2}}$ del problema de Dirichlet que le corresponde.

2. Se dice que el dominio $G_{2,3}$ satisface la condición de existencia de solución, si para cualquier función de frontera, continua sobre $\overline{\delta \cup \beta \cup \sigma}$, existe una solución continua en $\overline{G_{2,3}}$ del problema de Dirichlet que le corresponde.

3. Análogamente, se dice que el dominio $G=$ $G_{1,2} \cup G_{2,3}$ satisface la condición de existencia de solución, si para cualquier función de frontera, continua sobre $\overline{\alpha \cup \gamma \cup \sigma}$, existe una solución continua en $G$ del problema de Dirichlet correspondiente.

Teorema de Schwartz 1. Si el sistema de dominios $G_{1}$, $G_{2}$ y $G_{3}$ satisface el criterio de Schwartz y si para los dominios $G_{1,2}$ y $G_{2,3}$ se verifica la condición de existencia de solución, entonces esta misma condición se cumplirá también para el dominio compuesto $G=$ $G_{1,2} \cup G_{2,3}$.

Demostración. Supóngase que sobre $\overline{\alpha \cup \delta \cup \sigma}$ se ha fijado una función continua de frontera $f(s)$. Sea $g(x, y)$ alguna función continua en $G$ e igual a $f(s)$ sobre la frontera $\overline{\alpha \cup \delta \cup \sigma}$. Se afirma que existe un proceso iterativo que, partiendo de la aproximación inicial $\mathrm{g}(x, y)$, conduce a la solución del problema de Dirichlet.

En efecto, sea $\mu_{0}(x, y)=\mathrm{g}(x, y)$, se procede ahora a establecer las siguientes definiciones:

\section{Definición 2}

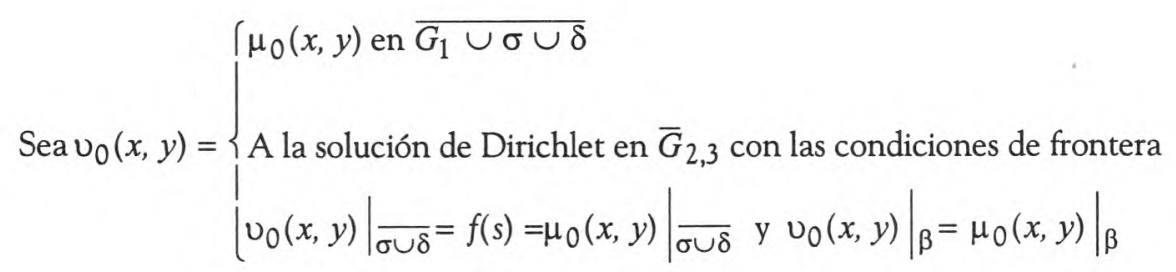

Las aproximaciones sucesivas $\mu_{n}(x, y)$ y $v_{n}(x, y)$ se definen de la siguiente manera:

\section{Definición 3}

$$
\mu_{n}(x, y)=\left\{\begin{array}{l}
\cup_{n-1}(x, y) \text { en } \overline{G_{3} \cup \sigma \cup \alpha} \\
\text { A la solución de Dirichlet en } \bar{G}_{1,2} \text { con las condiciones de frontera } \\
\left.\mu_{n}(x, y)\right|_{\overline{\sigma \cup \alpha}}=f(s)=\left.v_{n-1}(x, y)\right|_{\overline{\sigma \cup \alpha}} \text { y }\left.\mu_{n}(x, y)\right|_{\gamma}=\left.v_{n-1}(x, y)\right|_{\gamma}
\end{array}\right.
$$

Definición 4

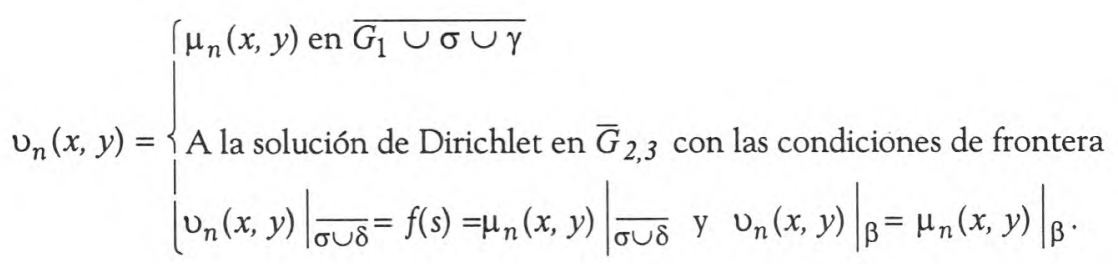


Es evidente que cada función $\mu_{n}(x, y)$ y $v_{n}(x, y)$ es continua en $\overline{\mathrm{G}}$, por su propia definición, y toma sobre la frontera los valores $f(s)$. Se demostrará que la sucesión

$$
\begin{gathered}
\mu_{0}(x, y), v_{0}(x, y), \mu_{1}(x, y), v_{1}(x, y), \mu_{2}(x, y), \ldots, \\
v_{\kappa-1}(x, y), \mu_{\kappa}(x, y), v_{\kappa}(x, y), \mu_{\kappa+1}(x, y) . .
\end{gathered}
$$

converge uniformemente. De aquí se seguirá a que su límite es una función continua que toma sobre la frontera $\alpha \cup \delta \cup \sigma$ los valores dados de $f(s)$.

Se afirma que la función límite es una función armónica en $G$.

En efecto, considérese algún punto $\left(x_{0}, y_{0}\right) \in \operatorname{int}(G)$.

Dicho punto será interior al menos para alguno de los dominios $G_{1,2} \circ G_{2,3}$.

Sin pérdida de generalidad, supóngase que $\left(x_{0}, y_{0}\right) \in G_{1,2}$. La sucesión

$$
\mu_{0}(x, y), \mu_{1}(x, y), \ldots, \mu_{n}(x, y), \ldots
$$

de funciones armónicas en $G_{1,2}$ también converge uniformemente. En virtud del teorema de Harnak ${ }^{1}$, su límite será una función armónica en $G_{1,2}$.

Si $\left(x_{0}, y_{0}\right)$ estuviese en el interior de $G_{2,3}$, razonando análogamente, habría que considerar la subsucesión $v_{0}(x, y), \cup_{1}(x, y), \cup_{2}(x, y), \ldots$ y establecer su límite armónico.

Luego, para concluir la verificación de la condición de existencia de la solución del problema de Dirichlet en $G$, es suficiente establecer la convergencia de la sucesión

$$
\mu_{0}(x, y), \cup_{0}(x, y), \mu_{1}(x, y), \cup_{1}(x, y), \mu_{2}(x, y), \ldots
$$

A tal propósito se observa que para $n=2,3,4, \ldots$

$$
\mu_{n}-\mu_{n-1}=\left\{\begin{array}{l}
v_{n-1}-v_{n-2} \text { en } \overline{G_{3} \cup \sigma \cup \alpha} \\
\text { A una función armónica en } G_{1,2} \text { igual a } \\
0 \text { en } \overline{\alpha \cup \sigma} \text { y a } \cup_{n-1}-v_{n-2} \text { en } \bar{\gamma}
\end{array}\right.
$$

Análogamente, para $n=1,2,3, \ldots$ se tiene

$$
v_{n}-v_{n-1}=\left\{\begin{array}{l}
\mu_{n}-\mu_{n-1} \text { en } \overline{G_{1} \cup \sigma \cup \delta} \\
\text { A una función armónica en } G_{2,3} \text { igual a } \\
0 \text { en } \overline{\delta \cup \sigma} \text { y a } \mu_{n}-\mu_{n-1} \text { en } \bar{\beta}
\end{array}\right.
$$

Sean ahora:

$$
b_{n}=\max _{(x, y) \in \bar{\gamma}}\left|v_{n}(x, y)-\mathrm{v}_{n-1}(x, y)\right| .
$$

Y dado que se satisface el criterio de Schwartz, se tiene que

$$
a_{n} \leq \theta b_{n-1} ; b_{n} \leq \theta a_{n} .
$$

De aquí se sigue que

$$
\begin{gathered}
a_{n} \leq \theta^{2} a_{n-1} \leq \theta^{2(n-2)} a_{2}, \\
b_{n} \leq \theta^{2 n-3} a_{2}(n \geq 2) .
\end{gathered}
$$

Por otra parte, el principio del máximo para la solución del problema de Dirichlet implica las desigualdades

$$
\begin{gathered}
\left|\mu_{n}(x, y)-\mu_{n-1}(x, y)\right| \leq b_{n-1} \leq \text { const } \mathrm{x} \theta^{2 n}, \\
\left|\mathrm{v}_{n}(x, y)-\mathrm{v}_{n-1}(x, y)\right| \leq a_{n} \leq \text { const } \mathrm{x} \theta^{2 n},
\end{gathered}
$$

$\operatorname{para}(x, y) \in \bar{G}$, y $n=2,3, \ldots$

La convergencia uniforme de las sucesiones

$$
\begin{gathered}
\mu_{0}, \mu_{1}, \mu_{2}, \ldots, \\
v_{0}, v_{1}, v_{2}, \ldots,
\end{gathered}
$$

se vuelve así evidente. Se observa que a partir de $n=1$, las funciones $v_{n}-\mu_{n}$ serán armónicas y continuas en cada uno de los recintos $G_{1}, G_{2}$ y $G_{3}$ y se anularán en la frontera exterior $\overline{\alpha \cup \delta \cup \sigma}$. A partir de la definición es inmediato que también

$$
v_{n}-v_{n-1}=0 \text { en } \bar{G}_{1} \text { y sobre } \beta \text {. }
$$

Sobre el arco $\bar{\gamma}$ la función $\mu_{n}$, por construcción, coincide con $v_{n-1}$. Por esto, $v_{n}-\mu_{n}$ es igual a $v_{n}-v_{n-1}$ sobre $\bar{\gamma}$. En virtud del principio del máximo

$$
\begin{gathered}
\left|v_{n}(x, y)-\mu_{n}(x, y)\right| \leq \max _{\bar{\gamma}}\left|v_{n}-\mu_{n}\right|= \\
\max _{\bar{\gamma}}\left|v_{n}-v_{n-1}\right|=b_{n} \times \text { const } \mathrm{x} \theta^{2 n} .
\end{gathered}
$$

$$
a_{n}=\max _{(x, y) \in \bar{\beta}}\left|\mu_{n}(x, y)-\mu_{n-1}(x, y)\right|,
$$

${ }^{1}$ En el apéndice se enuncia el teorema de Harnak. 
De aquí ya queda claro que la sucesión compuesta

$$
\mu_{0}(x, y), \mathrm{v}_{0}(x, y), \mu_{1}(x, y), \mathrm{v}_{1}(x, y), \mu_{2}(x, y), \mathrm{v}_{2}(x, y)
$$

también convergerá uniformemente.

\section{Generalización del método de Schwartz}

El método de Schwartz permite generalizar a dominios compuestos no solamente la existencia de la solución del problema de Dirichlet con valores de frontera continuos, sino también la validez del principio de Dirichlet ${ }^{2}$. Para tal fin se dará la siguiente definición:

Definición 5. La integral de Dirichlet en el dominio $G$ se especifica como:

$$
D_{G}(\mu)=\iint_{G}\left(\mu_{x}^{2}+\mu_{y}^{2}\right) d x d y
$$

Donde $\mu(x, y)$ es una función arbitraria y suficientemente lisa.

Se observa que la función $\mu(x, y)$ que figura en la definición 5 no necesariamente tiene que ser armónica.

Por otra parte, supongamos que la función $\mathrm{g}(x, y)$ es continua y lisa a trozos, con integral de Dirichlet finita $D_{G}(g)=D_{G_{1}}(g)+D_{G_{2}}(g)+D_{G_{3}}(g)$. Supóngase, asimismo, que el principio de Dirichlet se verifica para cada dominio $G_{1,2}, G_{2,3}$. Esto significa que para cualquier función $\mathrm{v}(x, y)$, lisa a trozos en $G_{1,2}$, y para una función $\mu(x, y)$ que sea armónica en dicho dominio y que coincida con $v$ sobre la frontera $\overline{\beta \cup \sigma \cup \delta}$, se verifica la desigualdad

$$
D_{G_{1,2}}(\mu) \leq D_{G_{1,2}}(\mathrm{U})
$$

En particular, para la sucesión de funciones $\mu_{n}, v_{n}$ utilizada en la demostración del teorema precedente, para $\mathrm{n}=1,2,3, \ldots$ se tiene

$$
D_{G_{1,2}}\left(\mu_{n}\right) \leq D_{G_{1,2}}\left(v_{n}\right)
$$

Se observa, además que $\mu_{n}=v_{n-1}$ en $G_{3}$; por lo tanto

$$
D_{G}\left(\mu_{n}\right) \leq D_{G}\left(\cup_{n-1}\right) .
$$

Uniendo las dos desigualdades anteriores se obtiene:

$$
D_{G}\left(\mu_{n}\right) \leq D_{G}\left(v_{n-1}\right), n=1,2,3, \ldots
$$

Análogamente, asumiendo que se cumple el principio de Dirichlet para $G_{2,3}$, no es difícil establecer la igualdad

$$
D_{G}\left(\mathrm{v}_{n}\right) \leq D_{G}\left(\mu_{n}\right), n=0,1,2, \ldots
$$

Como $\mu_{0}(x, y)=G$, se comprueba que la integral de Dirichlet de cualquiera de las funciones

$$
\mu_{0}, v_{0}, \mu_{1}, v_{1}, \mu_{2}, v_{2}, \ldots
$$

no supera a $D_{G}(g)$.Y se sabe que esta sucesión converge uniformemente a una función $\mu(x, y)$ armónica en $G$, que coincide con $g(x, y)$ sobre la frontera de dicho dominio.

Se afirma que

$$
D_{G}(\mu) \leq D_{G}(\mathrm{~g}) \text {. }
$$

En efecto, basta establecer la siguiente desigualdad:

$$
D_{H_{1} \cup H_{2} \cup H_{3}}(\mu)=D_{H_{1}}+D_{H_{2}}+D_{H_{3}} \leq D_{G}(g)
$$

para subdominios cualesquiera $H_{i}$ de las regiones

$$
G_{i}\left(H_{1} \subset G_{1}, H_{2} \subset G_{2}, H_{3} \subset G_{3}\right)
$$

y luego, recurrir a la igualdad

$$
D_{G}(\mu)=\sup D_{H_{1} \cup H_{2} \cup H_{3}}(\mu) .
$$

Dentro de cada subdominio $\mathrm{H}_{i}$, la sucesión

$$
\mu_{0}, v_{0}, \mu_{1}, v_{1}, \mu_{2}, v_{2}, \ldots
$$

estará formada por funciones armónicas y convergerá uniformemente junto con sus derivadas primeras. De aquí que

$$
D_{H_{1} \cup H_{2} \cup H_{3}}(\mu)=\lim _{n \rightarrow \infty} D_{H_{1} \cup H_{2} \cup H_{3}}\left(\mu_{n}\right) .
$$

Por otra parte, como

$$
D_{H_{1} \cup H_{2} \cup H_{3}}\left(\mu_{n}\right) \leq D_{G}\left(\mu_{n}\right) \leq D_{G}(g),
$$

se concluye la demostración de la desigualdad

$$
D_{G}(\mu) \leq D_{G}(g)
$$

\footnotetext{
${ }^{2}$ En el apéndice se enuncia el principio variacional de Dirichlet.
} 
Así pues, en virtud de la unicidad de la solución del problema de Dirichlet, la función $\mu(x, y)$ se determina unívocamente a partir de valores de frontera $\left.g(x, y)\right|_{\alpha \cup \delta \cup \sigma}$. Su existencia se sigue de la existencia de la solución del problema de Dirichlet, como ya se demostró, en el dominio $G=G_{1} \cup G_{2} \cup G_{3}$. La función que coincide con $g(x, y)$ sobre la frontera $\alpha \cup \delta \cup \sigma$ y que tiene integral de Dirichlet igual a $D_{G}(\mu)$ es única según el párrafo precedente.

\section{Discusión}

Se ha demostrado que del criterio de Schwartz para $G_{1}, G_{2}, G_{3}$ y de la validez del principio de Dichlet para $G_{1,2}$ y $G_{2,3}$, se sigue tanto la existencia de la solución del problema de Dirichlet, como la validez del principio de Dirichlet para la región $G=G_{1,2} \cup G_{2,3}$.

Por otra parte, se observa que en las demostraciones anteriores no era en absoluto relevante que los subdominios fuesen simplemente conexos, ni tampoco que cada arco de la frontera $\alpha, \beta, \gamma$ y $\delta$ fuese conexo. Luego cada uno de ellos puede estar formado por varios arcos diferentes, como por ejemplo, la gráfica que se muestra en la figura 2.

En esta figura el polígono doblemente conexo $G_{1} \cup G_{2} \cup G_{3}$ fue cortado, por medio de dos rectas paralelas $\beta(x=0)$ y $\gamma\left(\mathrm{x}=\frac{1}{2}\right)$, en varias partes. Los trozos $G_{1}^{1} \mathrm{y}$ $G_{1}^{2}$ son los componentes del dominio $G_{1}$. Los trozos $G_{1}^{1}, G_{2}^{2}$ y $G_{2}^{3}$ conforman al dominio $G_{2}$. Las fronteras $\alpha$ y $\delta$ están formadas, cada una, por tres poligonales $\alpha_{1}, \alpha_{2}, \alpha_{3}$ y $\delta 1, \delta 2, \delta 3$ respectivamente. La frontera $\sigma$ se divide en seis componentes distintos sobre cada una de las fronteras rectilíneas $\beta, \gamma$; deben considerarse tres segmentos; en la figura 3 se representan los dominios $G_{1}, G_{2}, G_{3}$ situados de manera que arco $\sigma$ no figura en absoluto.

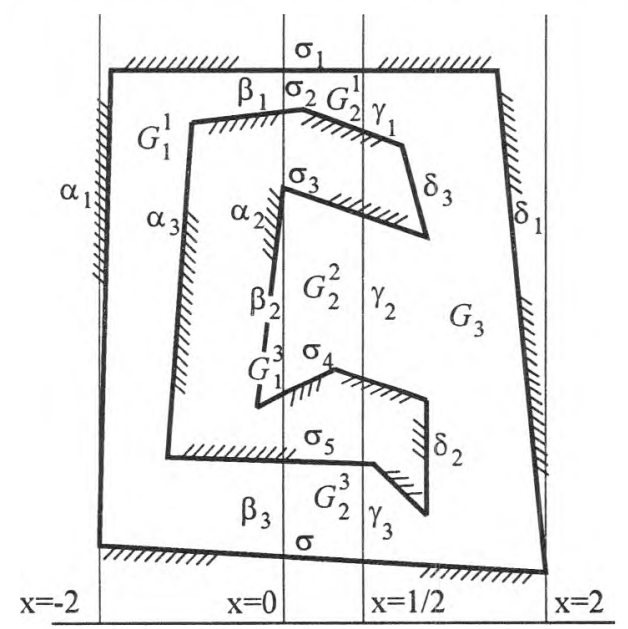

Figura 2. Subdominios no conexos
Se afirma que los dominios representados en la figura 2 satisfacen el criterio de Schwartz.

En efecto, sea la función armónica $\frac{2}{3}(x+2)$, no negativa en $G_{1} \cup G_{2}$ e igual a 1 sobre $\gamma\left(x=\frac{1}{2}\right)$. De su no negatividad, toda la figura se encuentra en la franja $-2 \leq x \leq 2$, tal y como puede apreciarse en la figura.

Es evidente que para cualquier función armónica continua que no supere a uno sobre $\gamma$, y se anule sobre $\alpha \cup$ $\sigma$, el pricipio el máximo implicará la desigualdad

$$
\mu(x, y) \leq \frac{2}{5}(x+2) .
$$

Si se sabe que $\mu(x, y)$ no es menor que -1 sobre $\gamma$ y se anula en $\alpha \cup \sigma$, análogamente se puede escribir que

$$
\mu(x, y) \geq-\frac{2}{5}(x+2) \text {. }
$$

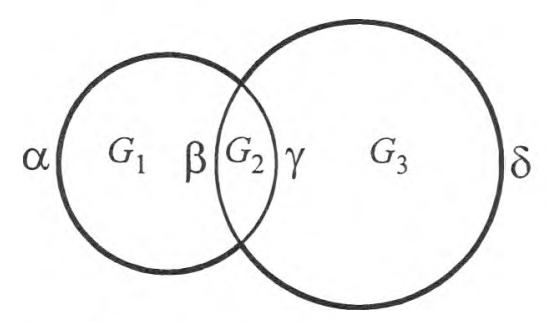

Figura 3. El dominio de la figura no contiene el arco $\sigma$

Es claro que si $|\mu(x, y)| \leq 1$ en $\gamma$ y $\mu=0$ sobre $\alpha \cup \sigma$, entonces para $x=0$, es decir, sobre $\beta$, tendrá lugar la desigualdad

$$
|\mu(x, y)| \leq \frac{2}{5}(x+2)=\frac{4}{5}
$$

De manera análoga, sólo que recurriendo a la función armónica $1-\frac{x}{2}$, positiva en $G_{1} \cup G_{2}$ e igual a uno sobre $\beta(\mathrm{x}=0)$, se muestra que cualquier función armónica que no supere a uno en valor absoluto sobre $\beta$ y se anule en $\delta \cup \sigma$, satisface sobre $\gamma(\mathrm{x}=$ $\frac{1}{2}$ ) la desigualdad $|\mu| \leq \frac{3}{4}$. Como se constata, en el caso considerado se verifica el criterio de Schwartz, si se escoge como $\theta$ :

$$
\theta=\frac{4}{5}=\operatorname{má} x\left(\frac{3}{4}, \frac{4}{5}\right)
$$

Si se hubiese fundamentado la existencia de solución del problema de Dirichlet y el principio de Dirichlet para los dominios simplemente conexos $G_{1}^{1} \cup G_{2}^{1}$ $\cup G_{2}^{3} \cup \beta_{1} \cup \beta_{3} G_{1}^{2} \cup G_{2}^{2} \cup \beta_{2}$ у $G_{3} \cup G_{2}^{1} \cup G_{2}^{2} \cup G_{2}^{3} \cup$ $\gamma_{1} \cup \gamma_{2} \cup \gamma_{3}$, entonces será válido generalizar estas propocisiones al dominio simplemente conexo $\overline{G_{1} \cup G_{2} \cup G_{3}}$. 
Obviamente, el razonamiento expuesto reviste carácter general.

\section{Algunos recursos geométricos}

Enseguida se procede a describir y fundamentar una condición geométrica muy sencilla y cómoda de verificar de la cual se seguirá la validez del principio de Schwartz.

Supóngase que dentro del dominio $G_{2}$ se puede escoger un subdominio $G_{2}^{2}$ delimitado por dos arcos de circunferencia que se intersequen en los puntos $A$ y $B$ ubicados en la frontera de $G_{2}$, de modo tal que los arcos $\beta$ y $\gamma$ queden en lados opuestos con respecto a la lúnula $A B$.

En la figura 4 se presentan algunas variantes admisibles de ubicación de la lúnula $G_{2}^{2}$ dentro del recinto $G_{2}$.
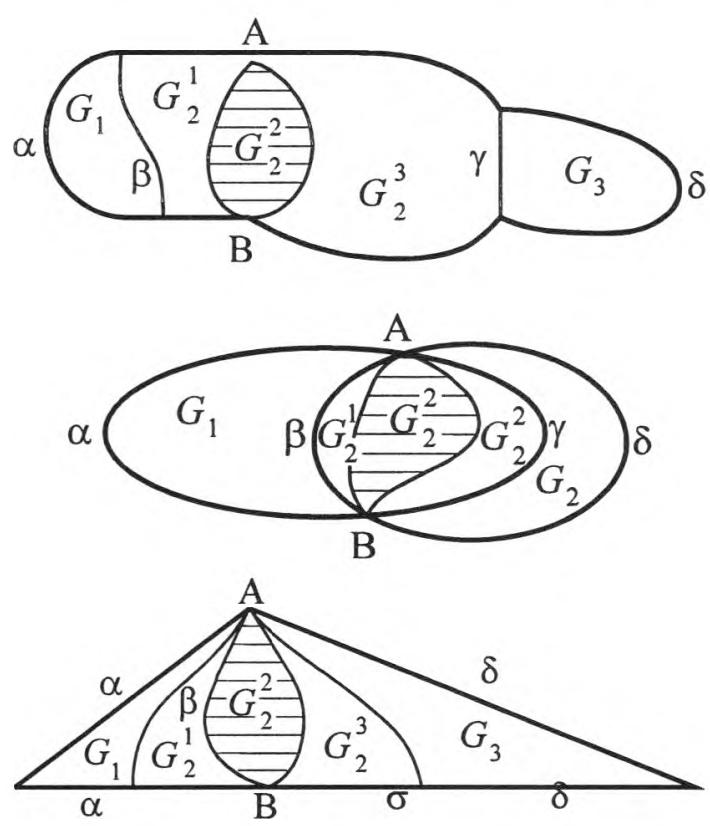

Figura 4. Algunas variantes admisibles de inserción de la lúnula

En cambio, en la figura 5 se representa un recinto dentro del cual resulta imposible ubicar una lúnula tal como se ha descrito, pues los arcos con $\beta$ y $\gamma$ son tangentes de los punto $P$ y $Q$.

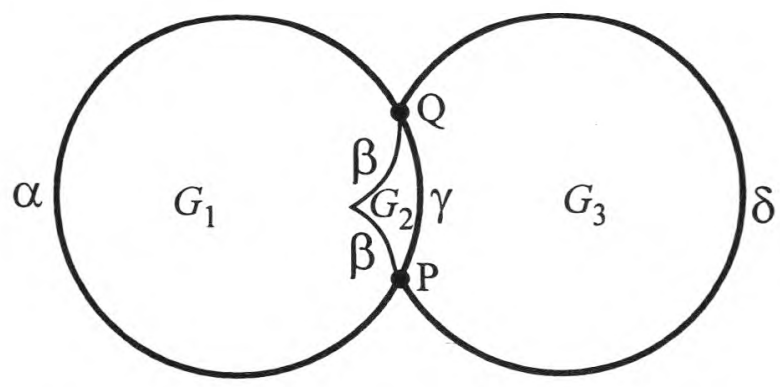

Figura 5. El dominio $G_{2}$ no admite inserción de la lúnula
Se afirma que si en $G_{2}$ se puede insertar una lúnula de la manera indicada, el criterio de Schwartz se verifica.

En efecto, supóngase que se conoce que alguna función armónica continua $\mu(x, y)$ es igual a cero sobre $\alpha \cup \sigma$ y no supera a uno en valor absoluto sobre $\gamma$. Entonces del principio del máximo se sigue que esta función no supera a uno en valor absoluto sobre el arco de circunferencia que delimita la lúnula por la derecha (Fig. 4).

Si se logra demostrar que el valor absoluto de la función en cuestión no es mayor que algún $\theta(\theta<1)$ sobre el arco izquierdo que delimita la lúnula, del principio del máximo se puede concluir sin dificultad que también sobre $\beta$ tendrá lugar la misma desigualdad. Es suficiente encontrar una función armónica $\omega(x, y)$ que sea igual a uno sobre el arco derecho, y no negativa en la parte restante de la frontera de la región $G_{1} \cup G_{2}^{1} \cup G_{2}^{2}$, para deducir, partiendo del principio del máximo, la desigualdad

$$
|\mu(x, y)| \leq \omega(x, y), \quad(x, y) \in G_{1} .
$$

Si además se tiene sobre el arco izquierdo de la lúnula $\omega(x, y)$ $\leq \theta$, entonces se habrá demostrado la proposición en cuestión.

A continuación se procede a construir una función armónica, mayorante de $\omega(x, y)$, uniforme en el plano con un corte a lo largo del arco derecho de la lúnula, que sea igual a uno sobre este arco a la izquierda del corte e igual a cero a su derecha. Se escribirá esta función de modo que sea constante sobre cada uno de los arcos de las circunferencias que pasen por los puntos $A, B$.

Con este fin, basta observar que la función

$$
\omega(x, y)=a\left(\operatorname{Arctg} \frac{y-y_{A}}{x-x_{A}}-\operatorname{Arctg} \frac{y-y_{B}}{x-x_{B}}+b\right)
$$

es armónica, y escoger las constantes $a$ y $b$, así como las determinaciones del arco tangente, las cuales deben satisfacer las condiciones impuestas. Sobre la frontera izquierda de la lúnula, la función $\omega$ tomará algún valor $\theta$, evidentemente positivo y menor que uno.

Ahora bien, no es posible utilizar la función $\omega(x, y)$ como mayorante con base en el principio del máximo (en su variante simple), pues ésta es discontinua en los puntos $A, B$ de la frontera de $G$. Sin embargo, esta función está acotada, lo cual permite aplicar el lema sobre la mayorante discontinua ${ }^{3}$.

De aquí, que de la igualdad $\mu(x, y)=0$ sobre $\alpha \cup \sigma$ y la desigualdad $\mu(x, y) \leq 1$ sobre $\gamma$ se sigue la desigualdad $|\mu|$ $\leq \theta$. La verificación de la primera mitad del criterio de Schwartz queda así concluida; la segunda mitad se verifica en forma totalmente análoga. 
Ahora se procede a mostrar cómo la aplicación del criterio de la lúnula permite comprobar fácilmente la existencia de la solución del problema de Dirichlet y la validez del principio de Dirichlet para culaquier polígono. Como se ha indicado anteriormente, el criterio en cuestión se limita a considerar únicamente polígonos simplemente conexos.

Supóngase que algún polígono puede ser cortado por medio de un segmento de recta $A B$, que se encuentra enteramente dentro de éste, en dos partes, $A P B$ y $B Q A$ (figura 6), para cada una de las cuales ya fue establecida la validez de lo que queremos probar.

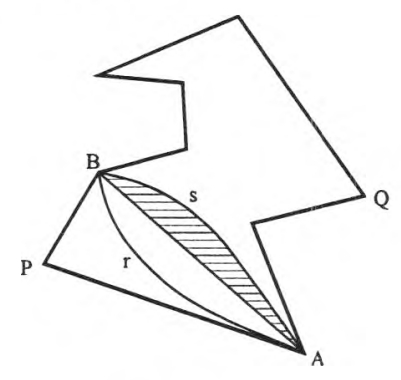

Figura 6.

Por otra parte, se procede a cubrir el segmento $A B$ por medio de alguna lúnula que se encuentre enteramente dentro del polígono. Como ya se ha demostrado en párrafo precedente, para la lúnula $A r B s$ se satisfacen tanto el principio de la existencia de la solución del problema de Dirichlet como del principio de Dirichlet. Así pues, recurriendo al criterio geométrico es fácil verificar también la existencia de solución y el principio de Dirichlet para la región $A P B S A$ formada por el polígono $A P B$, más la parte rayada de la lúnula.

Ahora bien, de la existencia de la solución del problema de Dirichlet y la satisfacción del principio de Dirichlet para $A P B S A$ y para $B Q A$ ya no es difícil concluir la validez de la existencia de la solución y del principio de dirichlet para $A P B Q A$.

Todo polígono simplemente conexo puede ser cortado por medio de segmentos de recta en triángulos de modo que cada corte aumente el número de dominios separados. Por esto bastará comprobar la existencia de la solución del problema de Dirichlet y la satisfacción del principio de Dirichlet para triángulos, inclusive para triángulos rectángulos (pues cualquier triángulo puede ser subdividido en dos triángulos rectángulos, por medio de alguna de sus alturas).

En la figura 7 se presenta la subdivisión del triángulo rectángulo $A B C$ en tres recintos $G_{1}, G_{2}, G_{3}$ por medio de los arcos $A E, A F$ de circunferencia que pasan por el punto $A$.

Es evidente que aquí también se verifica la condición de la lúnula y por ello resulta suficiente verificar la existencia de la solución del problema y del principio de Dirichlet únicamente para los sectores circulares $C A E, B A F$.

Pero al final de párrafo precedente ya fue probada la existencia de la solución del problema de Dichlet y la validez del principio de Dirchlet para sectores cualesquiera.

Así pues, a partir de cualquier función continua dada en la frontera de un polígono arbitrario con un número finito de lados, se asegura que dentro de éste existe una función armónica continua inclusive hasta la frontera y que coincide en la frontera con la función prefijada.

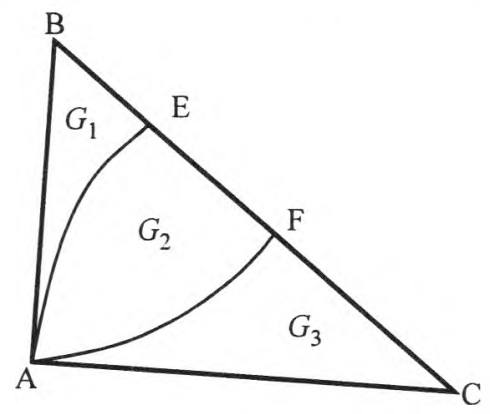

Figura 7. Subdivisión de un triángulo rectángulo

\section{Bibliografía}

Godunov S. K. (1984). Ecuaciones de la física matemática. Editorial Mir, Moscú.

Herrera I. (1994). Computional Mechanics Publications. Computational Methods in Water Resources XI, Southampton, Boston.

Herrera I. (1995). Domain Decomposition Methods for Model Parallelization. Second UNAM-Cray Supercomputing Conference in Numerical Simulation in the Environmental and Earth Sciences, México.

Hernández J., et al (1995). Domain Decomposition Methods to Flows. Second UNAM-Cray Supercomputing Conference in Numerical Simulation in the Environmental and Earth Sciences, México.

Herrera I. y Camacho A. (1996). A Non-Overlapping TH-Domain Decomposition. Invited Paper. Engineering Software, Vol. Domain Decomposition Methods.

\section{Apéndice}

Teorema de Harnak 1 . Sea $G_{\delta}$ el conjunto de puntos del dominio $G$ que se hallan a una distancia mayor que $\delta>0$ de su frontera, luego una sucesión de funciones armónicas continuas en un dominio $\bar{G}$ que converja uniformemente en la frontera $\Gamma$ del dominio $G$, será uniformemente convergente también dentro G; y su límite será una función armónica. Además, para cada subdominio $G_{\delta}$ interior, la

${ }^{3}$ En el apéndice se presenta el tema sobre la mayorante discontinua 
convergencia será uniforme no sólo para las propias funciones, sino también para las derivadas de cualquier orden.

Principio variacional de Dirichlet 1 . Sea $g(\rho, \vartheta)$ alguna función continua y lisa a trozos en el círculo $0 \leq \rho \leq R$, con alguna integral de Dirichlet finita

$$
D_{R} \int_{0}^{2 \pi} \int_{0}^{R}\left(g^{2}+\frac{1}{\rho^{2}} g_{\rho}\right) \rho d \rho d \vartheta
$$

Sobre la frontera del círculo $g(\rho, \vartheta)$ toma los valores de frontera

$$
f(\vartheta)=g(R, \vartheta)
$$

Considérese ahora la función armónica $\mu(\rho, \vartheta)$, que toma los mismos valores de frontera $\mu(R, \vartheta)=f(\vartheta)$.

Luego la integral de Dirichlet $\mathrm{D}_{R}(\mu)$ es finita y no supera a $D_{R}(g)$ :

$$
D_{R}(\mu) \leq D_{R}(g)
$$

Teorema de la mayorante discontinua 1. Sea $G$ un dominio acotado, con frontera $\Gamma$; se elige en $\bar{G}=\mathrm{G} \cup \Gamma$ un número finito de puntos $\left(x_{1}, y_{1}\right),\left(x_{2}, y_{2}\right) \ldots,\left(x_{N}\right.$, $\left.y_{N}\right)$. Algunos de ellos pueden estar dentro de $G$, otros sobre la frontera $\Gamma$. Sean $\mu(x, y), v(x, y)$ dos funciones continuas en $\mathrm{G} \cup \Gamma$ y armónicas en $\mathrm{G}$ con la posible excepción de los puntos $\left(x_{i}, y_{i}\right)$. En estos puntos, $\mu(x, y)$, $v(x, y)$ pueden tener discontinuidades o no estar definidas siquiera. Supóngase, sin embargo, que están acotadas:

$$
\begin{gathered}
|| \mu(x, y)|| \leq M, \\
|| \cup(x, y)|| \leq M .
\end{gathered}
$$

en $\mathrm{G} \cup \Gamma\left(\right.$ a excepción de los puntos $\left(x_{i}, y_{i}\right)$ ).

Si $\mu(x, y)|\Gamma \leq v(x, y)|_{\Gamma}$ en todos los puntos de la frontera, con la posible excepción de los puntos $\left(x_{i}, y_{i}\right)$, entonces será $\mu(x, y) \leq v(x, y)$ en todo $G \cup \Gamma($ nuevamente, con la posible excepción de los puntos $\left(x_{i}, y_{i}\right)$ ). 